\title{
Quality of life of African children with spina bifida: results of a validated instrument

\author{
Hanneke Jansen ${ }^{1}$, Esther Blokland ${ }^{1}$, Catharine de Jong*1, Janneke Greving ${ }^{2}$ \\ and Dan Poenaru ${ }^{3}$
}

\begin{abstract}
Address: ${ }^{1}$ University Medical Center Utrecht, the Netherlands KE 04133.1 P.P. Box 85090, 3508 AB Utrecht, the Netherlands, ${ }^{2}$ Julius Julius Center for Health Sciences and Primary Care, University Medical Center Utrecht, Utrecht, the Netherlands and ${ }^{3}$ BethanyKids at Kijabe Hospital AIC Kijabe hospital PO BOX 20 Kijabe, Kenya 00220, East Africa

Email: Catharine de Jong* - teaudejong@gmail.com

* Corresponding author
\end{abstract}

from 53rd Annual Meeting of the Society for Research into Hydrocephalus and Spina Bifida Belfast, UK. 24-27 June 2009

Published: 27 November 2009

Cerebrospinal Fluid Research 2009, 6(Suppl 2):S25 doi:10.1186/1743-8454-6-S2-S25

This abstract is available from: http://www.cerebrospinalfluidresearch.com/content/6/S2/S25

(c) 2009 Jansen et al; licensee BioMed Central Ltd.

\section{Background}

There are significant ethical issues in the management of children with spina bifida (SB), especially in resourcepoor settings. The arguments often center on quality of life (QoL) issues - yet there are no objective QoL studies from Africa to date. Our purpose was to determine QoL in these patients using a validated instrument, and correlate the QoL to the degree of symptoms and defects.

\section{Materials and methods}

SB children from Bethany Kids at Kijabe Hospital (BKKH) and its mobile clinics throughout Kenya were interviewed regarding their QoL using the validated Schedule for the Evaluation of Individual Quality of Life - Direct Weight (SEIQoL-DW). This instrument can transfer well across cultures by using open questions clustered in 6 domains chosen based on the study group responses. Symptoms and defects caused by SB were obtained from medical records. These included SB type, motor level, hydrocephalus, urinary incontinence, use of clean intermittent catheterization (CIC), and fecal incontinence. The same instrument was applied also to a group of age-matched healthy controls.

\section{Results}

The study included 166 children: 102 SB patients and 64 controls, aged between six months and 18 years. In 44 children the parents were also interviewed. The main defect was myelomeningocele $(68 \%), 59 \%$ had hydrocephalus, and CIC was used by $67 \%$ of 49 incontinent children older than 2.5 years. The SEIQoL score of children with SB was 65.1 (scale 0 - 100), as compared to 78 in the controls $(p<.001)$. SB children scored significantly lower than healthy controls in three domains; "health and disease", "development" and 'basic needs'; yet both groups identified "self-actualization" and "development" as the most important domains. There was no significant difference in the total SEIQOL scores whether the children or their parents were interviewed, and no single SB-related determinant made a significant difference in the score.

\section{Conclusion}

As expected, the QoL of African children with SB is lower than that of healthy controls, but remains surprisingly acceptable. No SB-related clinical factors appeared to influence significantly the QoL. The domains health and disease, development and basic needs differed significantly in the two groups. These domains need more attention in the future. 